In the format provided by the authors and unedited.

\title{
Dislocation nucleation facilitated by atomic segregation
}

Lianfeng Zou ${ }^{1}$, Chaoming Yang ${ }^{2}$, Yinkai Lei ${ }^{3}$, Dmitri Zakharov ${ }^{4}$, Jörg M.K. Wiezorek ${ }^{3}$, Dong Su${ }^{4}$, Qiyue Yin ${ }^{1}$, Jonathan $\mathrm{Li}^{5}$, Zhenyu Liu ${ }^{3}$, Eric A. Stach ${ }^{4}$, Judith C Yang ${ }^{6}$, Liang Qi ${ }^{2}$, Guofeng Wang ${ }^{3}$, Guangwen Zhou ${ }^{1 *}$

${ }^{1}$ Department of Mechanical Engineering \& Materials Science and Engineering Program, State University of New York at Binghamton, NY 13902

${ }^{2}$ Department of Materials Science and Engineering, University of Michigan, Ann Arbor, MI 48109

${ }^{3}$ Department of Mechanical Engineering and Materials Science, University of Pittsburgh, Pittsburgh, PA 15261

${ }^{4}$ Center for Functional Nanomaterials, Brookhaven National Laboratory, Upton, NY 11973

${ }^{5}$ Department of Physics, Applied Physics and Astronomy \& Materials Science and Engineering Program, State University of New York, Binghamton, NY 13902

${ }^{6}$ Department of Chemical and Petroleum Engineering, University of Pittsburgh, Pittsburgh, PA 15261

*Correspondence to: gzhou@binghamton.edu

This file includes:

Extended sections 1 to 14

Extended Data Tables 1-2

Extended Data Figures 1 to 14

Captions for Supplemental Videos 1 to 7

References

Other Supplementary Materials for this manuscript includes the following: Supplemental Videos 1 to 7 


\section{Extended Data Section 1. Edge thinning under the strong electron beam condition (Extended Data} Figures 1 and 2)

Edge thinning effects can be observed in the environmental TEM (ETEM) experiments with a strong electron beam current and in STEM experiments with a condensed beam probe. Extended data Figs. 1(a-b) show a typical example of the edge thinning along the (110) facet while we deliberately used a strong beam to focus on the edge area, which resulted in the edge thinning along the (110) edge, as shown by the significantly weakened image intensity of the characteristic Au-segregation induced trough-crest surface reconstruction. Meanwhile, the electron-beam induced edge thinning led to the formation of a new (110) ledge that is three atomic layers away from the old one. The Au segregation along the newly formed (110) ledge resulted in the same characteristic trough-crest surface reconstruction, as shown in the HRTEM image (Extended data Fig.1b). This indicates that the intense beam illumination can facilitate the knock off of atoms from the edge area and lead to the edge thinning. However, the edge thinning effect is negligible when we spread the e-beam, which was the typical operation condition that we used for capturing the (110) surface dynamics in the TEM model shown in the manuscript.

In the STEM model, the beam effect could be more significant because of the higher probe current density and longer acquisition time required for STEM imaging. The e-beam induced edge thinning effect can be also seen from the STEM image (Extended data Fig.1c), where the scanning process along the (110) facet knocked out some surface atomic columns, and the remaining surface columns are still visible, as pointed by the white arrows.

We also acquired HAADF images with various detector collection angles by changing the camera length to check for any variation in image contrast. For example, we collected images using the inner angles of $54 \mathrm{mrad}, 68 \mathrm{mrad}$ and $90 \mathrm{mrad}$, which correspond to the camera length of $10 \mathrm{~cm}, 8 \mathrm{~cm}$ and $6 \mathrm{~cm}$, respectively. All of the STEM images consistently showed the edge thinning effect (please see Extended Fig. 2), which allowed us to rule out any potential effect from the collection angle induced surface contrast changes. We also compared the STEM images acquired at $350{ }^{\circ} \mathrm{C}$ and room temperature, both of which showed the characteristic trough-crest $\mathrm{Cu}_{3} \mathrm{Au}$ surface contrast without visible contrast difference 
on the topmost layer, indicating the negligible temperature effect (i.e., the Debye-Waller effect) on the image contrast.

\section{Extended Data Section 2. HRTEM image simulations (Extended Data Figure 3)}

The formation of faceted holes in the $\mathrm{Cu}-\mathrm{Au}$ thin films during the oxide removal annealing may result in some edge thinning relative to the original bulk thin film thickness. We performed HRTEM image simulations to ascertain the origin of the atomic column contrast difference between the surface region and the bulk. The simulated HRTEM images obtained using the $\mathrm{Cu}_{3} \mathrm{Au}$ segregated configuration consistently reproduced the characteristically darker $\mathrm{Au}$ atom column contrast for the surface region relative to the $\mathrm{Cu}(\mathrm{Au})$ alloy lattice of the bulk region (Fig. 1e). On the contrary, the simulated HRTEM images obtained using model crystals with a reduced thickness at the outermost surface (Extended data Fig. 3), considering the effect of edge thinning, produced more uniform contrast of the atom columns in both the surface and bulk alloy regions, which is inconsistent with the experimental observations. The HRTEM image contrast simulations further confirm that the experimentally observed $\{100\}$-type nanofacets of the trough-crest reconstructed (110) surface are attributed to the $\mathrm{Cu}_{3} \mathrm{Au}$ segregated configuration.

\section{Extended Data Section 3. DFT simulation (Extended Data Figure 4)}

The reconstructed (110) surface of the $\mathrm{Cu}_{90} \mathrm{Au}_{10}$ alloy was modeled by a slab with one bulk terminated (110) surface and one $(2 \times 1)$ reconstructed (110) surface as shown in Extended data Fig. 4. 15 atomic layers were used in all models with 58 atoms in total. The trough-crest reconstructed (110) surface in the TEM image shows ordering similar to the (110) surface of $\mathrm{Cu}_{3} \mathrm{Au}$. Therefore, three possible terminations of the reconstructed (110) surface of $\mathrm{Cu}_{3} \mathrm{Au}$ were used on the reconstructed surface of the slab (Extended data Figs. 4(b-d)). Moreover, a model with randomly distributed Au atoms was built as a reference (Extended data Fig. 4e). In all these models, the number of Au atoms in bulk was adjusted in order to keep an overall Au concentration of 10.3 at. \% (6 Au atoms). Besides, the bottom 5 layers of the super cells were fixed in all the models (see Extended data Fig. 4a). The lattice constant (3.759 $\AA$ ) was 
determined by a linear interpolation between the lattice constant of pure $\mathrm{Cu}(3.622 \AA)$ and $\mathrm{Au}(4.175 \AA)$. All models were relaxed until the Hellman-Feynman force exerted on each atom was less than $0.01 \mathrm{eV} / \AA$. The stability of each possible termination was measured by the energy difference between the corresponding model and the random model. We found that the energies of the models in Extended data Figs. $4 \mathrm{~b}$ and $4 \mathrm{~d}$ are lower than the energy of the random model by $302.2 \mathrm{~mJ} / \mathrm{m}^{2}$ and $145.6 \mathrm{~mJ} / \mathrm{m}^{2}$, respectively. On the contrary, the energy of the structure shown in Extended data Fig. 4c is $239.8 \mathrm{~mJ} / \mathrm{m}^{2}$ higher than the energy of the random model (Extended data Fig. 4e). Therefore, the $\mathrm{Cu}_{3} \mathrm{Au}$ type termination of ordering as shown in extended data Fig. $4 \mathrm{~b}$ is predicted to be the most energetically favorable termination among the four modeled terminations on the reconstructed (110) surface of $\mathrm{Cu}_{90} \mathrm{Au}_{10}$.

\section{Extended Data Section 4. Calculation of the critical thickness of the coherent $\mathrm{Cu}_{3} \mathrm{Au}$ segregated} layer

According to the classical Matthews-Blakeslee model for the onset of misfit dislocations in thin films on the bulk substrate, the interfacial misfit dislocations can be generated if the thickness of the thin films is above a critical value $h_{c}$. Corrected for a single array of misfit dislocations in two dimensions, $h_{c}$ can be obtained by numerically solving the following equation ${ }^{1}$

$$
h_{c}=\frac{b}{8 \pi(1+v) f}\left[\ln \left(\frac{h_{c}}{b}\right)+1\right] \text {. }
$$

Here $\mathrm{v}$ is Poisson ratio and $\mathrm{v}=1 / 3$; $\mathrm{b}$ is the length of Burgers vector and it equals to $\frac{\sqrt{2}}{2} \times a_{C u_{3} A u}=2.65 \AA$ $\left(a_{C u_{3} A u}=3.75 \AA\right)$; $\mathrm{f}$ is the misfit strain that can vary on the scale of $2 \sim 3 \%$ depending on the local Au concentrations. By numerically solving the above equation, we can find that the critical thickness varies from $3.43 \AA$ to $8.62 \AA$ with the strain changing from $2.9 \%$ to $2.0 \%$.

\section{Extended Data Section 5. In-situ TEM visualization of the decay of pairs of atomic columns of Au} from the (110) surface (Extended Data Figure 5) 

the [020] direction. Therefore, there are a total of four columns of surface atoms that have peeled off from the circled region shown in Figs. 2(a-b) during the time interval of $0.5 \mathrm{~s}$, which results in a locally deepened trough (four atomic layers deep).

\section{Extended Data Section 7. DFT modeling of the stability of surface atoms (Extended Data Figure 7)}

The physical reason for the departure of four columns of surface atoms can be related to the locally insufficient Au segregation, for which the surface tends to develop locally a deeper trench for initiating dislocation nucleation (i.e., Fig. 2). To address this question, we employed DFT to calculate the stability 
129

130

131

132

133

134

135

136

137

138

139

140

141

142

143

144

145

146

147

148

149

of the surface atoms. Extended Data Figure 7 shows the surface configuration of the $\mathrm{L}_{2}$ ordered $\mathrm{Cu}_{3} \mathrm{Au}$ segregated layer based on our experiment result (e.g., Figs. 1 and 2). We then calculated the vacancy formation energy by removing each of the atoms (labeled 1, 2, 3 and 4) in the four columns of surface atoms. The DFT results (see Extended Data Table 1) show that the energy cost for removal of the outermost atom (atom 1) is $0.28 \mathrm{eV}$. After atom 1 has been removed, the energy barrier for the removal of atom 2 is only $0.07 \mathrm{eV}$. This is a strong indication that atom 2 becomes highly unstable once losing the protection from the outermost $\mathrm{Au}$ atom (i.e., atom 1) and is thus prone to diffuse away together with atom 1 instead of detaching individually. This is consistent with our in-situ TEM observation that shows that surface atoms detach from the surface in pairs of $\mathrm{Cu}$ and $\mathrm{Au}$ atoms (please see Supplementary in-situ TEM video 1).

The departure of surface pairs (atoms 1 and 2) leads to the exposure of very unstable surface sites (atom 3). The energy cost for vacancy formation at site 3 is $-0.33 \mathrm{eV}$, which indicates that the removal of atom 3 is a spontaneous process. The further removal of atom 4 is slightly less that the removal of the Au atom at site 1 . However, if $\mathrm{Au}$ surface segregation is sufficiently fast to replace the $\mathrm{Cu}$ atom at site 3 during the decay of the surface pair of atoms 1 and 2, this can improve the stability of the $\mathrm{Cu}$ and Au pair at sites 3 and 4 . Therefore, the reason for the departure of more surface atoms from a localized region can be attributed to the stochastic process of locally insufficient Au segregation, for which the exposed $\mathrm{Cu}$ atoms are more vulnerable to decay from the surface, thereby developing into a locally deeper trench.

Extended Data Table 1: DFT computed vacancy formation energies for removing atoms 1-4 labeled in Extended data Fig.7

\begin{tabular}{|c|c|}
\hline & \\
Site & Vacancy Formation Energy (eV) \\
\hline 1 & 0.28 \\
\hline 2 & 0.07 \\
\hline 3 & -0.33 \\
\hline 4 & 0.25 \\
\hline
\end{tabular}


150

151

152

153

154

155

156

157

158

159

160

161

162

163

164

165

166

167

168

169

170

171

172

173

174

Extended Data Section 8. Locate dislocation positions from IFFT and identify the core structure of the dislocations in the parent $\mathrm{Cu}(\mathrm{Au})$ region (Extended Data Figure 8)

HRTEM image simulations are used to identify the core structure of the dislocations in the parent $\mathrm{Cu}(\mathrm{Au})$ region using different configurations of the dislocation lines. Extended data Figs. 8(d-h) show the simulated HRTEM images obtained from several representative structure models including three configurations with different inclined dislocation lines and one example with a screw component for the unit dislocations with line direction [001]. Their comparison shows that the one with the pure edge component $\left(\mathbf{b}=\frac{1}{2} a[\overline{1} 10](110)\right.$ provides the best match with the experimental HRTEM images, where the dislocation core only affect the contrast of a very localized area, whereas the other core structures affect the image contrast of a larger area.

\section{Extended Data Section 9. Random walk characteristic of dislocation cores (see Extended Data}

\section{Figure 9)}

The migration trajectories can be tracked after the dislocations move into the parent $\mathrm{Cu}(\mathrm{Au})$ area. Extended data Figs. 9(a-d) depict a sequence of in-situ TEM images showing the migration path of a misfit dislocation in the parent $\mathrm{Cu}(\mathrm{Au})$ region. Extended data Fig. 9(e) shows the distance of the dislocation from its initial location as a function of time. At first, the dislocation is observed to oscillate at a stand-off distance of about $4 \sim 6 \mathrm{~d}_{220}$ lattice spacings (i.e., a distance of $1 \mathrm{~nm}$ to $1.5 \mathrm{~nm}$ from the $\mathrm{Cu}_{3} \mathrm{Au} / \mathrm{Cu}(\mathrm{Au})$ interface). It is surprising to note from the in-situ TEM observations (Supplementary insitu TEM videos 6 and 7) that the motion by glide and climb is of similar probability once the dislocation fluctuates at a stable distance, which is the typical feature of a random walk. To confirm the nature of the random walk of the dislocation motion, the mean displacements of the dislocation core are obtained from frame-by-frame measurements on individual images of the in-situ TEM video. In our analysis, 40 timesequential TEM images were used. For each time interval (i.e., $\Delta \mathrm{t}=1 \mathrm{~s}, 2 \mathrm{~s}, 3 \mathrm{~s}$, etc.), there are 20 displacements used for averaging. For example, the 20 displacements for $\Delta t=1 \mathrm{~s}$ are those between $\mathrm{t}_{1}=0$ 
175

176

177

178

179

180

181

182

183

184

185

186

187

188

189

190

191

192

193

194

195

196

$\mathrm{s}$ and $\mathrm{t}_{2}=1 \mathrm{~s}, \mathrm{t}_{2}=1 \mathrm{~s}$ and $\mathrm{t}_{3}=2 \mathrm{~s}, \mathrm{t}_{3}=2 \mathrm{~s}$ and $\mathrm{t}_{4}=3 \mathrm{~s}$, etc.; the 20 displacements for $\Delta \mathrm{t}=2 \mathrm{~s}$ are those between $t_{1}=0 \mathrm{~s}$ and $t_{3}=2 \mathrm{~s} ; \mathrm{t}_{2}=1 \mathrm{~s}$ and $\mathrm{t}_{4}=3 \mathrm{~s}, \mathrm{t}_{3}=2 \mathrm{~s}$ and $\mathrm{t}_{5}=4 \mathrm{~s}$, etc., where $\mathrm{t}_{1}, \mathrm{t}_{2}, \mathrm{t}_{3}, \ldots$, correspond to the time-sequence TEM images. The starting location of the dislocation is given in the first TEM image (i.e., $\mathrm{t}_{1}=0 \mathrm{~s}$ ). The mean square displacements (MSD) $\overline{\mathrm{d}}^{2}$ for each time interval $\Delta \mathrm{t}$ is calculated using the following equation,

$$
\bar{d}^{2}(\Delta t)=\frac{1}{40-\Delta t+1} \sum_{t=0}^{40-\Delta t}[\vec{r}(t+\Delta t)-\vec{r}(t)]^{2},
$$

where $\vec{r}(t)$ is the displacement vector of the dislocation at time t with respect to the moment $(\mathrm{t}-\Delta \mathrm{t})$. Therefore, the calculated results $\bar{d}^{2}$ represent the average over different sets of motions. As shown in extended data Fig. 9(f), the MSD of the dislocation core increases linearly with time, indicative of a standard random walk characteristic. The error bars in Extended data Fig. 9(f) are the uncertainties $e(\Delta t)$ calculated based on the variation of the square displacements at each time interval, which can be defined as the square root of the quotient of the variation by the number of data points, as described by the following equation,

$$
e(\Delta t)=\sqrt{\frac{\overline{d^{4}}(\Delta t)-\left[\overline{d^{2}}(\Delta t)\right]^{2}}{n(\Delta t)-1}},
$$

where $\overline{d^{4}}(\Delta t)$ is the average of fourth power of the displacement, $\overline{d^{2}}(\Delta t)$ is the mean square displacement and $n(\Delta t)$ is the number of data points at $\Delta t$. Note that there are no error bars in Extended data Fig. 9(e) because there is only one displacement for each point. The diffusivity of the dislocation core is estimated to be $0.27 \AA^{2} / \mathrm{s}$ with an uncertainty of $\pm 0.03 \AA^{2} / \mathrm{s}$.

\section{Extended Data Section 10. DFT calculation of GSF curve and APB energy (Extended Data Figure}

10)

We apply density functional theory (DFT) calculations to obtain the generalized stacking fault (GSF) energy along the $[\overline{1} 10](110)$ slip system as well as the $1 / 2[\overline{1} 10](110)$ antiphase boundary energy of 
$197 \mathrm{~L}_{2} \mathrm{Cu}_{3} \mathrm{Au}$. The DFT calculation is performed on $\mathrm{VASP}^{2}$ with non-spin-polarized conditions. The 198 pseudopotentials of $\mathrm{Cu}$ and $\mathrm{Au}$ are obtained by the projector augmented wave method $(\mathrm{PAW})^{3}$ with the 199 Perdew-Burke-Ernzerhof exchange-correlation functional (PBE) ${ }^{4}$. We take the first-order MethfesselPaxton smearing of $0.4 \mathrm{eV}$ for partial occupancies of each orbital and kinetic cutoff energy of $360.00 \mathrm{eV}$ throughout the calculation.

In the calculation of the GSF along [1110](110), our calculation cells take X, Y, Z base vectors along the direction of [110], [001] and [110], respectively. For pure $\mathrm{Cu}$, we take the $(2.6 \AA \times 3.7 \AA \times$ $31.4 \AA$ ) supercell of 20 atoms and mesh the K-points by $(18 \times 18 \times 1)$ with the Monkhorst-Pack scheme. We also construct the $(5.2 \AA \times 7.4 \AA \times 31.4 \AA)$ supercell of $79 \mathrm{Cu}$ atoms and $1 \mathrm{Au}$ atom to calculate the GSF along $[\overline{1} 10](110)$ under the Au alloying effect, where the Au atom is located on the (110) layer nearest to the glide interface (Extended data Fig. 10(a)). The K-points are the $(11 \times 11 \times 1)$ MonkhorstPack mesh. We implement the selective dynamics scheme such that all atoms are allowed to move only along the [110] direction during relaxation, which is perpendicular to the (110) glide interface. We sample ten equidistance configurations as shifting the (110) plane of the supercell along [110] to calculate the energy and perform cubic spline interpolation in order to obtain the GSF curve of the (110) plane glide along the $[\overline{1} 10]$. Our calculation shows that the Au solute on (110) layer nearest to the glide interface increases the unstable stacking fault along $[\overline{1} 10](110)$ by $\sim 50 \mathrm{meV} / \mathrm{m}^{2}$. In addition, we extend our calculations of GSF for all possible vectors on the (110) slip plane, which shows no local minimum of GSF energies except those corresponding to the full Burgers vector of $1 / 2[\overline{1} 10]$ in either pure $\mathrm{Cu}$ and $\mathrm{Cu}(\mathrm{Au})$ alloys, which suggests no stable configurations of partial dislocation Burgers vectors on this slip plane.

In calculation of the APB along (110) of the $\mathrm{L}_{2} \mathrm{Cu}_{3} \mathrm{Au}$ structure, we build the ordered $\mathrm{L}_{2}$ $\mathrm{Cu}_{3} \mathrm{Au}$ supercell of $36 \mathrm{Cu}$ atoms and $12 \mathrm{Au}$ atoms, with the supercell size of $(5.7 \AA \times 4.1 \AA \times 40.2 \AA)$ (extended data Fig. 10(b)). The basic Cartesian vectors are chosen along [110], [001], [110], respectively, and K-points are meshed by $(21 \times 21 \times 1)$ to ensure convergence. We add $1 / 2[\overline{1} 10]$ shift on half of the $(110)$ 
222

223

224

225

226

227

228

229

230

231

232

233

234

235

236

237

238

239

240

241

242

243

244

245

246

planes of the supercell to generate the APB configuration and calculate the energy of the supercell with and without APB. The DFT results show that the APB energy along the (110) plane of $\mathrm{L}_{2} \mathrm{Cu}_{3} \mathrm{Au}$ is $0.1097 \mathrm{eV} / \mathrm{A}^{2}$

\section{Extended Data Section 11. Blocking effect of the $\mathrm{L1}_{2} \mathrm{Cu}_{3} \mathrm{Au}$ segregated layer on dislocation penetration (see Extended Data Figure 11)}

The initial configuration of the $1 / 2[\overline{1} 10]$ edge dislocation is generated by displacing atoms according to the continuum displacement field of edge dislocations ${ }^{5}$ with static minimization of the system energy. According to the size of the experimental samples, we take the $56 \mathrm{~nm} \times 44 \mathrm{~nm} \times 3 \mathrm{~nm}$ simulation box of 463000 atoms, with the fixed boundary condition along $\mathrm{x}$ and $\mathrm{y}$ directions and periodic boundary condition along the z-direction. All of the potentials employed in calculations of the nearsurface $1 / 2[1 \overline{1} 0]$ edge dislocation provide well-consistent result that the dislocations without the APB barrier annihilate on the free surface in pure $\mathrm{Cu}$ within several picoseconds by local diffusionless transformation (Extended data Figs. 11(b, d)).

In addition, via calculations of two different configurations of surface atomistic structures (i.e., the perfect $(1 \times 1)$ surface and the experimentally observed $(2 \times 1)$ reconstruction), we verify that the dislocation is dragged to the free surface and annihilate regardless the different surface configurations for pure $\mathrm{Cu}$. We estimate the energy decrement by calculating the energy difference before and after the dislocation annihilation on the free surface, and find the energy decreases by $0.685 \mathrm{eV} / \AA$ in the case of the $(2 \times 1)$ reconstructed surface and $0.343 \mathrm{eV} / \AA$ for the non-reconstructed surface.

To be consistent with the experimental observations that the near-surface dislocations can exist for several seconds and even much longer time, we construct three surface layers of $\mathrm{L}_{2} \mathrm{Cu}_{3} \mathrm{Au}$ with the (2×1) reconstruction on the bulk substrate with randomly 10 atomic percent of $\mathrm{Au}$ in the $\mathrm{Cu}$ lattice. We also introduce the $1 / 2[\overline{1} 10](110)$ edge dislocation in the bulk slightly below the ordered $\mathrm{L}_{2} \mathrm{Cu}_{3} \mathrm{Au}$ surface layer. By tuning the initial position of the dislocation and running MD simulation for $\sim 10$ nanoseconds at 
247

248

249

250

251

252

253

254

255

256

257

258

259

260

261

262

263

264

265

266

267

268

269

270

271

$600 \mathrm{~K}$, we verify that the dislocation glides along the $\mathrm{Cu}_{3} \mathrm{Au} / \mathrm{Cu}(\mathrm{Au})$ interface without entering the $\mathrm{Cu}_{3} \mathrm{Au}$ segregated layer (Extended Data Fig. 11(a)). By ignoring the chemical effect of Au atoms, we perform the same calculation on the $\mathrm{Cu}-\mathrm{Ag}$ system based on the $\mathrm{Cu}-\mathrm{Ag}$ EAM potential ${ }^{6}$. We introduce three layers of the $\mathrm{L}_{2}-\mathrm{Cu}_{3} \mathrm{Ag}$ as the segregated surface layer on the bulk substrate with randomly 10 atomic percent of $\mathrm{Ag}$ atoms in $\mathrm{Cu}$ lattice. The MD simulation still shows the dislocation glide along the $\mathrm{L}_{2}-\mathrm{Cu}_{3} \mathrm{Ag} / \mathrm{Cu}(\mathrm{Ag})$ interface without penetrating through the $\mathrm{Cu}_{3} \mathrm{Ag}$ layer (Extended data Fig. 11(c)), indicating that the stability of near-surface dislocations is significantly enhanced by the presence of the chemically ordered structure of the segregated layer.

In the simulation of the formation of APB, we modify the initial position of the $1 / 2[\overline{1} 10](110)$ edge dislocation to generate the dislocation within the $\mathrm{L1}_{2}$-ordered $\mathrm{Cu}_{3} \mathrm{Au}$ segregated layer, then run $\mathrm{MD}$ for 100 picoseconds (Figs. 4(c-d)). After the energy minimization, we find that the system energy increases by $\sim 21 \mathrm{eV}$ with the presence of an APB, which illustrates that the large energy barrier for the APB formation prevents the dislocation from entering the $\mathrm{Cu}_{3} \mathrm{Au}$ segregated layer. In fact, the energy increase due to APB is proportional to the total APB area. Because the energy decrease due to surface dislocation annihilation is on the scale of $0.5 \sim 1 \mathrm{eV} / \AA$ from the above MD simulations, and the APB energy cost is on the scale of $0.1 \mathrm{eV} / \mathrm{A}^{2}$ from first-principles calculations in Extended data section 11, it means that the APB can prevent the dislocation annihilation as long as its length along the direction perpendicular to the dislocation line ([110] in our cases) is about 5 10 $\AA$, which is easy to be achieved in the thin film samples in our experiments.

In verification of the mobility of the $1 / 2[\overline{1} 10]$ edge dislocation on the (110) slip plane, we use MD simulations to examine the critical resolved shear stress (CRSS) to drive the $1 / 2[\overline{1} 10](110)$ dislocation glide in pure $\mathrm{Cu}$ at $630 \mathrm{~K}$. We take the $(36 \times 25 \times 22 \mathrm{~nm})$ simulation box of 1152000 atoms to decrease the pinning effect generated by the periodicity along the $\mathrm{z}$ direction. By adding shear strain on the (110) plane at the strain rate of $4.75 \times 10^{6}$ per second, we find the CRSS of the $1 / 2[110](110)$ dislocation of $\mathrm{Cu}$ at $630 \mathrm{~K}$ is $\sim 11 \mathrm{Mpa}$, which is generated by $1.55 \times 10^{-4}$ of shear strain. 

the system energy. On the other hand, dislocations easily migrate to the surface for both the perfect and reconstructed (110) surfaces for pure $\mathrm{Cu}$ (Extended Data Figure 11). These results further confirm that that it is the development of the $\mathrm{Cu} 3 \mathrm{Au}$ surface alloy to stabilize the sub-surface dislocations and their subsequent migration is dominated by the interplay between the composition fluctuations and the image force.

280

Extended Data Section 12. Investigations on the near-surface dislocation behavior using the continuum elastic theory and its comparison with MD simulations (Extended Data Figures 11(e, f) and Extended Data Table 2)

\section{A: Stress distribution due to surface image effect}

To calculate the stress on the near-surface dislocation, we first construct the image dislocation whose stress distribution cancels parts of the initial edge dislocation (Extended data Fig. 11(e)). With the image, the only stress left along the surface equals

$$
\sigma_{x y}(x, y=l)=2 \frac{\mu b}{2 \pi(1-v)} \frac{x\left(x^{2}-l^{2}\right)}{\left(x^{2}+l^{2}\right)^{2}}
$$
given by Eq. 1. To solve the biharmonic equation,

$$
\frac{\partial^{4} \phi}{\partial x^{4}}+2 \frac{\partial^{4} \phi}{\partial x^{2} \partial y^{2}}+\frac{\partial^{4} \phi}{\partial y^{4}}=\nabla^{4} \phi=0
$$
$\phi=X(x) Y(y)$ such that

$$
\frac{\partial^{4} Y}{\partial y^{4}}+\frac{2}{X} \frac{\partial^{2} X}{\partial x^{2}} \frac{\partial^{4} Y}{\partial y^{2}}+\frac{Y}{X} \frac{\partial^{4} X}{\partial x^{4}}=0
$$


295

296

297

298

299

300

301

302

303

304

305

which has the solution $X=\alpha \cos (k x)+\beta \sin (k x)$, where $k$ is a free parameter. Therefore, the biharmonic equation becomes

$$
\frac{\partial^{4} Y}{\partial y^{4}}-2 k^{2} \frac{\partial^{2} Y}{\partial y^{2}}+k^{4} Y=0
$$

which has the solution $Y=(a+b y) e^{k y}+(c+d y) e^{-k y}$. We then consider the boundary conditions as $y \rightarrow \infty, Y=0$, therefore, $a=b=0$, and $Y(y=0, x)=0$ gives $c=0$, thus $Y=d y e^{-k y}$. We also notice that the derivative of the Airy stress respective to $x$ produces an odd function, thus, we drop the $\sin (k x)$ term in $X(x)$. Furthermore, since the parameter $\mathrm{k}$ can be any value to satisfy the equation, based on the sum rule, the solution is given by

$$
\phi=\int_{0}^{\infty} \alpha(k) y e^{-k y} \cos (k x) d k
$$

The boundary condition on the free surface generates

$$
\left(\frac{\partial^{2} \phi}{\partial x \partial y}\right)_{y=0}=-\sigma_{x y}(x, y=0)=-\int_{0}^{\infty} \alpha(k) k \sin (k x) d k
$$

$$
=\frac{\mu b}{\pi(1-v)} \frac{x\left(x^{2}-l^{2}\right)}{\left(x^{2}+l^{2}\right)^{2}}
$$

It is the form of Fourier Integral formula, thus

$$
\alpha(k) k=-\frac{\mu b}{\pi^{2}(1-v)} \int_{-\infty}^{\infty} \frac{x\left(x^{2}-l^{2}\right)}{\left(x^{2}+l^{2}\right)^{2}} \sin (k x) d x .
$$

We decrease the order of $x$ via integration by parts, then utilize the residue theorem to integrate. After one step of integration by parts, Eq. 7 becomes

$$
\alpha(k) k=\frac{\mu b}{\pi^{2}(1-v)} \frac{1}{k} \int_{-\infty}^{\infty} \frac{x^{4}-6 x^{2} l^{2}+l^{4}}{\left(x^{2}+l^{2}\right)^{3}} \cos (k x) d x
$$

By using the residue theorem, we finally obtain 


$$
\alpha(k)=\frac{\mu b}{\pi(1-v)}\left(l-\frac{1}{k}\right) e^{-k l}
$$

$$
\phi=\frac{\mu b}{\pi(1-v)}\left[\frac{l y(l+y)}{(l+y)^{2}+x^{2}}-\int_{0}^{\infty} \frac{1}{k} e^{-k(y+l)} y \cos (k x) d k\right]
$$

314 We calculate the force that attracts the dislocation toward the free surface as the following: based on the

315 Peach-Koehler equation, $\vec{F}=(\sigma \vec{b}) \times \vec{\xi}$, where the $\sigma, \vec{b}$ and $\vec{\xi}$ are the stress matrix, the Burgers vector and the unit dislocation line vector, respectively. The force acting on the dislocation is

$317 \vec{F}=\left[F_{x}, F_{y}, F_{z}\right]=\left[\sigma_{x y} b, \sigma_{x x} b, 0\right]$, where $\sigma_{x y}$ and $\sigma_{x x}$ are stress components and $b$ is norm of the Burgers satisfies

$$
\sigma_{x x}=\frac{\partial^{2} \phi}{\partial y^{2}}
$$

and substituting $\phi$ by (10), we have

$$
\begin{gathered}
\sigma_{x x}=\frac{\mu b}{\pi(1-v)}\left\{\frac{2 l\left(x^{4}-3 x^{2} y(l+y)-l(l+y)^{3}\right)}{\left[x^{2}+(l+y)^{2}\right]^{3}}+\right. \\
\left.\frac{2(l+y)^{3}+2(l+y) x^{2}-y(l+y)^{2}+y x^{2}}{\left[(l+y)^{2}+x^{2}\right]^{2}}\right\}
\end{gathered}
$$

such that

$$
\sigma_{x x}(x=0, y=l)=\frac{\mu b}{\pi(1-v)} \frac{1}{2 l}
$$

The stress generated by the image dislocation is

$$
\sigma_{x x}^{i}=-\frac{\mu b}{2 \pi(1-v)} \frac{1}{2 l}
$$

Therefore, the stress exerted on the dislocation by the free surface is

$$
\sigma_{x x}^{s}=\sigma_{x x}+\sigma_{x x}^{i}=\frac{\mu b}{4 \pi(1-v)} \frac{1}{l}
$$


329

330

331

332

333

334

335

336

337

338

339

340

341

342

343

344

345

346

347

348

which drags the dislocation to the free surface.

\section{B: Equilibrium due to the coherent strain and the image force}

The coherent strain due to the lattice mismatch at the interface will generate the coherent stress, which also drives the dislocation climb. The strain along the y direction (direction perpendicular to (110) surface) is ignored since there is no lattice misfit involved along the y direction. Following the same plain strain condition assumption and isotropic elasticity, we obtain the coherent stress

$$
\sigma_{x x}^{c}=\frac{2 \mu v}{1-2 v}\left(\varepsilon_{x x}+\varepsilon_{z z}\right)+2 \mu \varepsilon_{x x}
$$

Hence, the total stress $\sigma_{x x}^{t}$ is the sum of the stress from the free surface and the stress due to the lattice misfit as the following,

$$
\sigma_{x x}^{t}=\sigma_{x x}^{s}+\sigma_{x x}^{c}
$$

$$
=\frac{\mu b}{\pi(1-v)} \frac{1}{4 l}+\frac{2 \mu \nu}{1-2 v}\left(\varepsilon_{x x}+\varepsilon_{z z}\right)+2 \mu \varepsilon_{x x}
$$

We can use the elastic constants of pure $\mathrm{Cu}(17)$ as the approximation to the $\mathrm{Cu}_{90} \mathrm{Au}_{10}$ solid solution, i.e., $\mu \sim 70-76 \mathrm{GPa}$, and $v \sim 0.41-0.42$. For the $1 / 2[110](110)$ edge dislocation, $\mathrm{b}=\sqrt{2} / 2 a$. Thus, by solving the stress equilibrium equation $\sigma_{x x}^{t}=0$, the equilibrium distance of the dislocation to the surface is described as the following,

$$
l_{c}=\frac{1}{4 \pi(1-v)\left(\frac{2}{1-2 v}\left(\varepsilon_{x x}+\varepsilon_{z z}\right)+2 \varepsilon_{x x}\right)} b
$$

The equilibrium distance sits in the range of $\sim 2 \AA$ to $20 \AA$ as the lattice strain varies from $2.0 \%$ to $0.2 \%$. The equilibrium distance is well consistent with our experimental observations in Fig. 3.

\section{C: Image dragging force and the energy for surface dislocation annihilation}



Based on the Peach-Koehler equation, the dragging force due to surface image effect acting on the dislocation can be written as

$$
F_{y}=\varepsilon_{x x} b=\frac{\mu b^{2}}{4 \pi(1-v)} \frac{1}{l} .
$$

Therefore, the work done by dragging the dislocation is

$$
\begin{aligned}
W & =\int_{l_{1}}^{l_{2}} F_{y}(x) d l=\int_{l_{1}}^{l_{2}} \frac{\mu b^{2}}{4 \pi(1-v)} \frac{1}{l} d l \\
& =\frac{\mu b^{2}}{4 \pi(1-v)}\left[\log \left(l_{1}\right)-\log \left(l_{2}\right)\right]
\end{aligned}
$$

The work in Eq. 20 is the elastic energy variation for the near-surface dislocation climbing from a location at $l_{1}$ below the surface to another location at $l_{2}$ below the surface. If $l_{2}$ is very close to the surface (comparable to the dislocation core radius), the work in Eq. 20 can be regarded as the energy variation for the near-surface dislocation annihilation if the core contribution to the energy variation is ignored. Thus, to verify the accuracy of our model, we compare the energy variation calculated in Eq. 20 and the energy variation from MD simulations of the near-surface dislocation annihilation shown in Fig. 4A (also illustrated in Extended Data Figure 11 (b) and (d)).

Based on our MD simulations of pure Cu in Fig. 4A (also in Extended Data Figures 11(b, d)), the initial distance from the dislocation core to surface $1_{1}$ is 3 atomic layers $(\sim 7.6 \AA)$. We define a cutoff distance $\mathrm{dc}$ as the final and closest distance that the dislocation can reach to the surface before its annihilation. According to Eq. 20, we can calculate the work by the dragging force on the dislocation located at $l_{1}$ below the surface to $l_{2}=d_{\mathrm{c}}$, where $d_{\mathrm{c}}$ is comparable with the radius of the dislocation core (Extended Data Table 2). Our MD simulations show that the energy drop due to the dislocation annihilation on the free surface is $0.685 \mathrm{eV} / \AA$ and $0.343 \mathrm{eV} / \AA$, respectively, in the two different (110) surface configurations, i.e., the perfect $(1 \times 1)$ surface and the $(2 \times 1)$ reconstructed surface, as mentioned in Section S11 (also shown in Extended Data Figures 11(b, d)). According to Extended Data Figure 11 and our MD simulations, the minimum $l_{2}$ that a dislocation can reach to the top surface before annihilation is 
371 about $1 \sim 2 \AA$ for the perfect surface and $\sim 3 \AA$ the $(2 \times 1)$ reconstructed surface, so the elastic energy

372 variation calculated by Eq. 20 with $l_{1}=7.6 \AA$ to $l_{2}=1.5 \AA$ corresponds to the energy drop from MD

373 simulations for the perfect surface, and the elastic energy variation calculated with $l_{1}=7.6 \AA$ to $l_{2}=3.0 \AA$

374 corresponds to the energy drop from MD simulations for the reconstructed surface. As shown in Extended

375 Data Table 2 and Extended Data Figure 11(f), the results based on the continuum elastic theory and our

376 MD simulations agree with each other very well for both perfect surface and reconstructed surface cases.

Extended data Table 2: The work by dragging the dislocation from $l_{1}$ below the free surface to $l_{2}=$

\begin{tabular}{|l|l|l|l|}
\hline Cutoff $\mathrm{d}_{\mathrm{c}}[\AA]$ & 1.0 & 2.0 & 3.0 \\
\hline Energy $[\mathrm{eV} / \AA]$ & 0.8506 & 0.5606 & 0.3909 \\
\hline
\end{tabular}

\section{Extended Data Section 13. Negligible beam effect on surface restructuring and dislocation motion}

To further check any potential e-beam effects on possible surface restructuring (i.e., to rule them 
394

395

396

397

398

399

400

401

402

403

404

405

406

407

408

409

410

411

412

413

414

415

416

417

418

Fig. 12(a)). The thin film was then annealed at $350{ }^{\circ} \mathrm{C}$ and 0.001 Torr of $\mathrm{H}_{2}$ gas flow to produce holes in the film while the beam was blanked off during the annealing process. After $\sim 20$ min of annealing, a hole formed in the film when the e-beam was un-blanked for TEM imaging. Extended Data Figure 12(c) illustrates a HRTEM image from the hole edge area, which shows that the characteristic Au-segregation induced trough-crest surface reconstruction of the $\mathrm{Cu}_{3} \mathrm{Au}$ surface layer already developed along the (110) facet of the hole edge (where the focusing and crystal zone-axis orientation were performed on an adjacent region of the specimen). These comparison experiments confirm the negligible effect of the electron beam irradiation on the Au-segregation induced formation of the trough-crest reconstructed surface $\mathrm{Cu}_{3} \mathrm{Au}$ layer. Conducting experiments of this type to understand the effect of electron irradiation is part of our experimental protocol, and in this case ensured that electron irradiation has a negligible effect on the observed surface evolution.

To rule out any electron beam effect from the in-situ TEM observation on dislocation motion, we first identified a dislocation that has already migrated into the bulk (Extended Data Figure 13(a)). The ebeam was blanked off for a few seconds and then un-blanked for TEM imaging, which showed that the dislocation has undergone some migration by moving toward the surface side by a few atomic spacings without the e-beam irradiation (Extended Data Figure 13(b)). Such TEM observation confirms that the electron beam effect has a negligible effect on the dislocation motion and the dislocation migration that we see are inherent.

\section{Extended Data Section 14. Formation of misfit dislocations by Au surface segregation at $600{ }^{\circ} \mathrm{C}$} (Extended Data Figure 14)

$\mathrm{Au}$ surface segregation induced dislocation formation was also observed by annealing the $\mathrm{Cu}_{90} \mathrm{Au}_{10}$ sample at $600{ }^{\circ} \mathrm{C}$ and $1 \times 10^{-3}$ Torr of $\mathrm{H}_{2}$ gas flow show. Extended data Figs. $14(\mathrm{a}, \mathrm{b})$ are the HRTEM images of the surfaces after $\mathrm{Au}$ segregation. Rather than forming the $\mathrm{Cu}_{3} \mathrm{Au}$ segregated surface layer at $350{ }^{\circ} \mathrm{C}$, one can note that annealing the sample at $600{ }^{\circ} \mathrm{C}$ results in the surface segregation of $\mathrm{Au}$ 
419

420

421

422

423

424

425

426

427

428

429

430

431

432

433

434

435 atoms that further aggregate as Au clusters on both the (100) and (110) surfaces. This is because the $\mathrm{Cu}_{3} \mathrm{Au}$ ordered phase is stable only up to $390{ }^{\circ} \mathrm{C}$ and the higher annealing temperature significantly promotes the atom mobility for the surface segregation and clustering of Au atoms. Clearly, the HRTEM images show the formation of misfit dislocations at the $\mathrm{Au} / \mathrm{Cu}(\mathrm{Au})$ interface. The observed dislocation arrays along both the [100] and [110] directions indicates there is a dislocation network at the $\mathrm{Au} / \mathrm{Cu}(\mathrm{Au})$ interface.

It should be noted that the small magnitude of in-plane misfit strain between the lattices of the $\mathrm{Cu}(\mathrm{Au})$ alloy substrate and the segregation layer of $\mathrm{Cu}_{3} \mathrm{Au}, \sim 2.7 \%$, can be accommodated elastically and a coherent interfacial structure can be retained under the conditions of the in-situ E-TEM experiments. This permits for mobility of the newly formed interfacial dislocations during the experimental observations. For larger magnitudes of interfacial misfit between the substrate alloy and the segregation facilitated surface layer phase a reduced mobility and transition to a semi-coherent interface structure with formation of a misfit dislocation array can be expected (e.g., at $\mathrm{T}=600^{\circ} \mathrm{C}$, Au forms in the segregation layer with $\sim 10-11 \%$ lattice misfit and a misfit dislocation array formation would be energetically favorable, and the misfit dislocations should be quite immobile, since they can only accommodate misfit strain while resident in the interface as well as the strong dislocation-dislocation elastic interactions and dislocation line entanglements in the dislocation network. 
436

437

438

439

440

441

442

443

444

445

446

447

448

449

450

451

452

453

454

455

456

457

458

459

460

461

\section{Captions for Supplementary in-situ TEM videos}

Supplemental in-situ TEM video 1: In-situ TEM video showing the peeling off of pairs of atomic columns from the (110) surface

Supplemental in-situ TEM video 2: In-situ TEM video showing the birth of a misfit dislocation at the $\mathrm{Cu}_{3} \mathrm{Au} / \mathrm{Cu}(\mathrm{Au})$ interface via a surface trapping process

Supplemental in-situ TEM video 3: In-situ TEM video showing the nucleation of a misfit dislocation at the $\mathrm{Cu}_{3} \mathrm{Au} / \mathrm{Cu}(\mathrm{Au})$ interface and its subsequent glide along the $\mathrm{Au} / \mathrm{Cu}(\mathrm{Au})$ interface

Supplemental in-situ TEM video 4: In-situ TEM video showing the dislocation migration by glide toward the outer (110) surface, which results in the dislocation annihilation at the outer surface

Supplemental in-situ TEM video 5: In-situ TEM video showing the dislocation migration by positive climb into the parent $\mathrm{Cu}(\mathrm{Au})$ region

Supplemental in-situ TEM video 6: In-situ TEM video showing the random walk of a dislocation in the parent $\mathrm{Cu}(\mathrm{Au})$ region

Supplemental in-situ TEM video 7: In-situ TEM video showing the behavior of a dislocation in the subsurface region during the retraction of a trough-crest reconstructed $\mathrm{Cu}_{3} \mathrm{Au}(110)$ ledge on the planar (100) surface

2
3

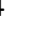


462

463

464

465

466

467

468

469

470

471

472

473

474

475

476

477

478

479

480

481

482

483

484

485

486

487

488

489

\section{References}

1. L. Dong, J. Schnitker, R. W. Smith, D. J. Srolovitz, Stress relaxation and misfit dislocation nucleation in the growth of misfitting films: A molecular dynamics simulation study. J. Appl. Phys. 83, 217-227 (1998).

2. G. Kresse, J. Furthmüller, Software VASP, vienna (1999). Phys. Rev. B 54, 169 (1996).

3. G. Kresse, D. Joubert, From ultrasoft pseudopotentials to the projector augmented-wave method. Phys. Rev. B. 59, 1758 (1999).

4. J. P. Perdew et al., Erratum: Atoms, molecules, solids, and surfaces: Applications of the generalized gradient approximation for exchange and correlation. Phys. Rev. B. 48, 4978 (1993).

5. J. P. Hirth, J. Lothe, Theory of dislocations. (Krieger Publishing Company 1982).

6. P. Williams, Y. Mishin, J. Hamilton, An embedded-atom potential for the $\mathrm{Cu}-\mathrm{Ag}$ system. Model. Simul. Mater. Sci. Eng. 14, 817 (2006). 

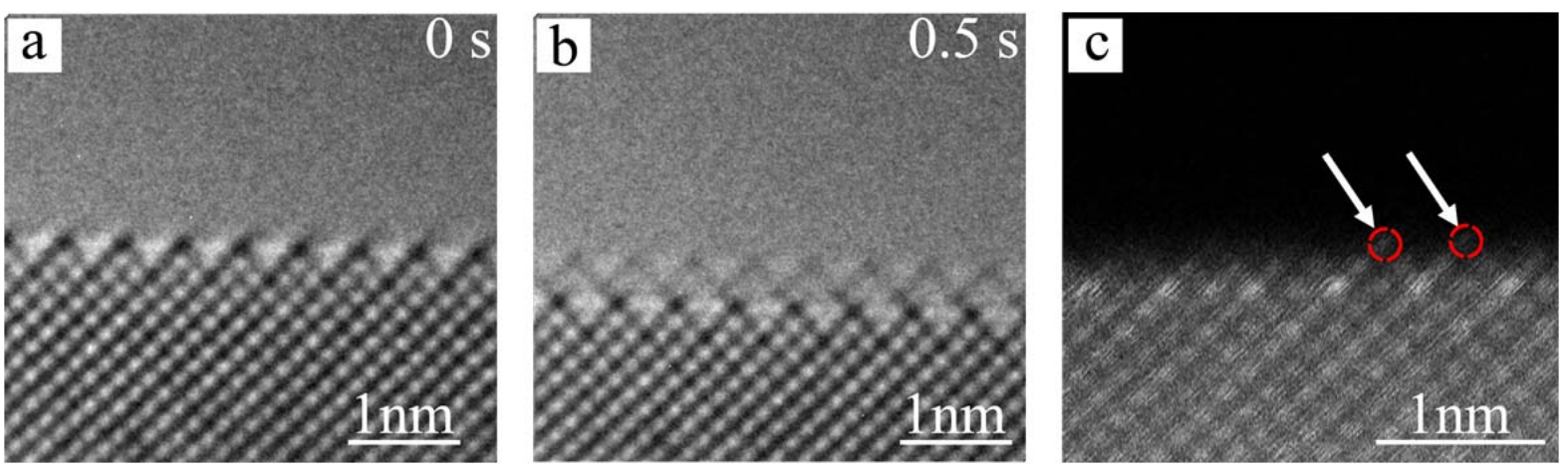

Extended Data Figure 1 | E-TEM and STEM imaging of the e-beam induced edge thinning effect. a, HRTEM image showing the trough-crest $\mathrm{Cu}_{3} \mathrm{Au}$ surface. b. HRTEM image showing the edge thinning effect by deliberately using a strong beam to focus on the edge area. The e-beam induced edge thinning led to the formation of a new (110) ledge that is three atomic layer away from the outmost (110) edge and the Au-segregation along the newly formed (110) ledge resulted in the characteristic trough-crest surface reconstruction. c, STEM image of the Au-segregation induced trough-crest surface reconstruction. The white arrows point to the remaining atomic columns on the topmost layer (circled by the dash red rings), while the rest of the atomic columns on the topmost layer were knocked during the e-beam scanning process. 

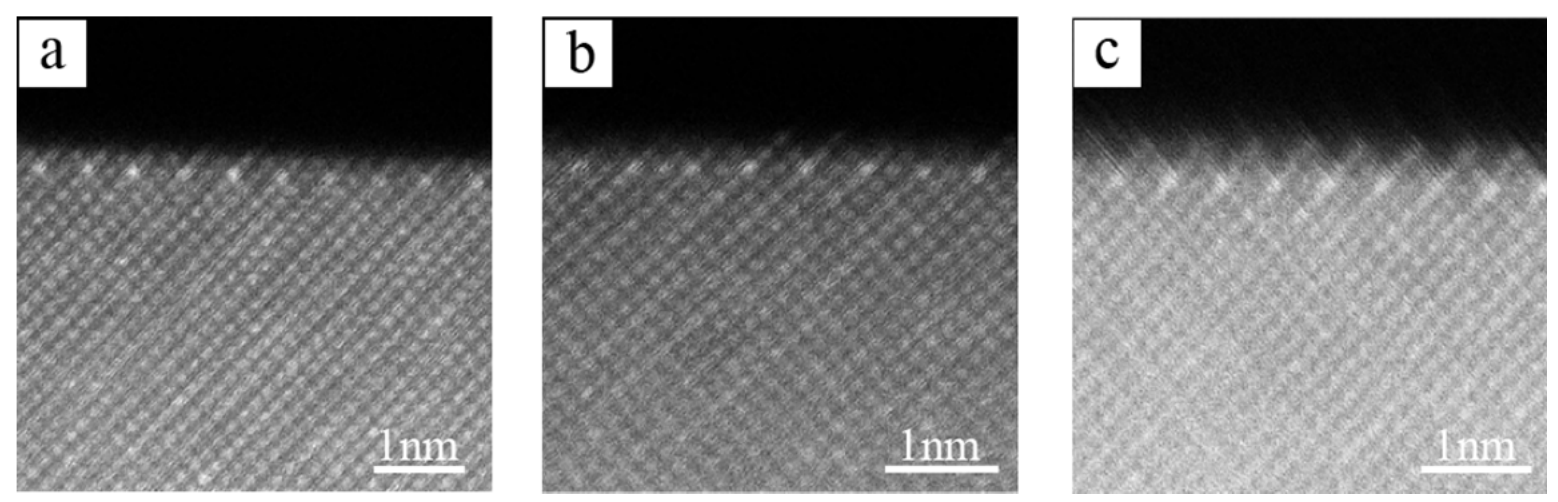

Extended Data Figure 2 HAADF STEM images of the (110) surface with different inner collection angles. a-c, the inner collection angles at $90 \mathrm{mrad}, 68 \mathrm{mrad}$ and $54 \mathrm{mrad}$ by using the camera length of 6 $\mathrm{cm}, 8 \mathrm{~cm}$ and $10 \mathrm{~cm}$, respectively. The outermost atomic layer shows a weaker contrast than the inner atomic layers in all the three imaging conditions, indicating that the weakened contrast in the outermost atomic layer is induced by the STEM condensed electron beam effect that resulted in some edge thinning

509 relative to the bulk thin film region. 

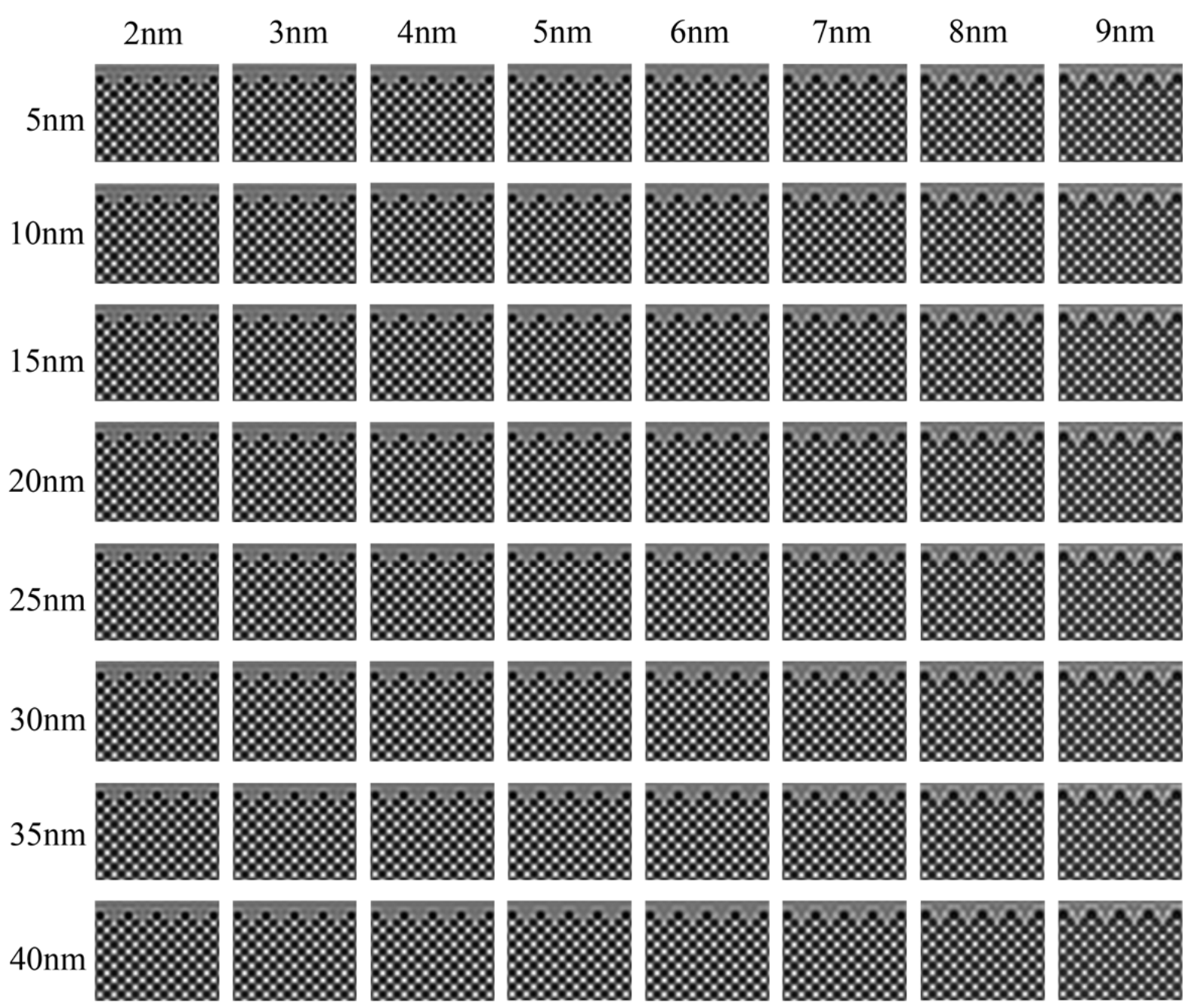

511
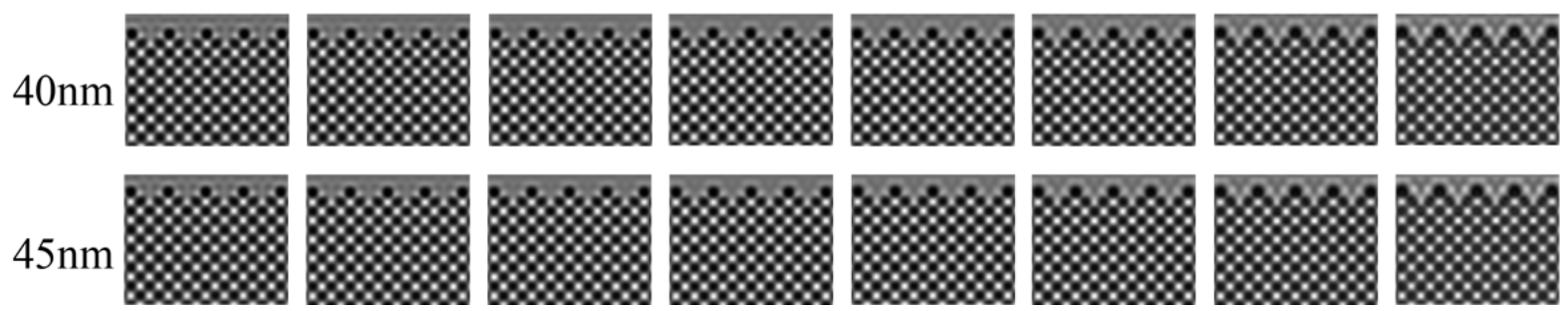

512 Extended Data Figure $3 \mid$ Calculated HRTEM images of the trough-crest reconstructed $\mathrm{Cu}_{90} \mathrm{Au}_{10}$ surface

513 alloy. The specimen has a bulk thickness of $50 \mathrm{~nm}$ (starting from the second outmost layer) with the

514 thickness of the topmost layer increasing from $5 \mathrm{~nm}$ to $45 \mathrm{~nm}$ (from top to bottom). The defocus value

515 increases from $2 \mathrm{~nm}$ to $9 \mathrm{~nm}$ (from left to right).

516

517

518 
520

521

522

523

524

525
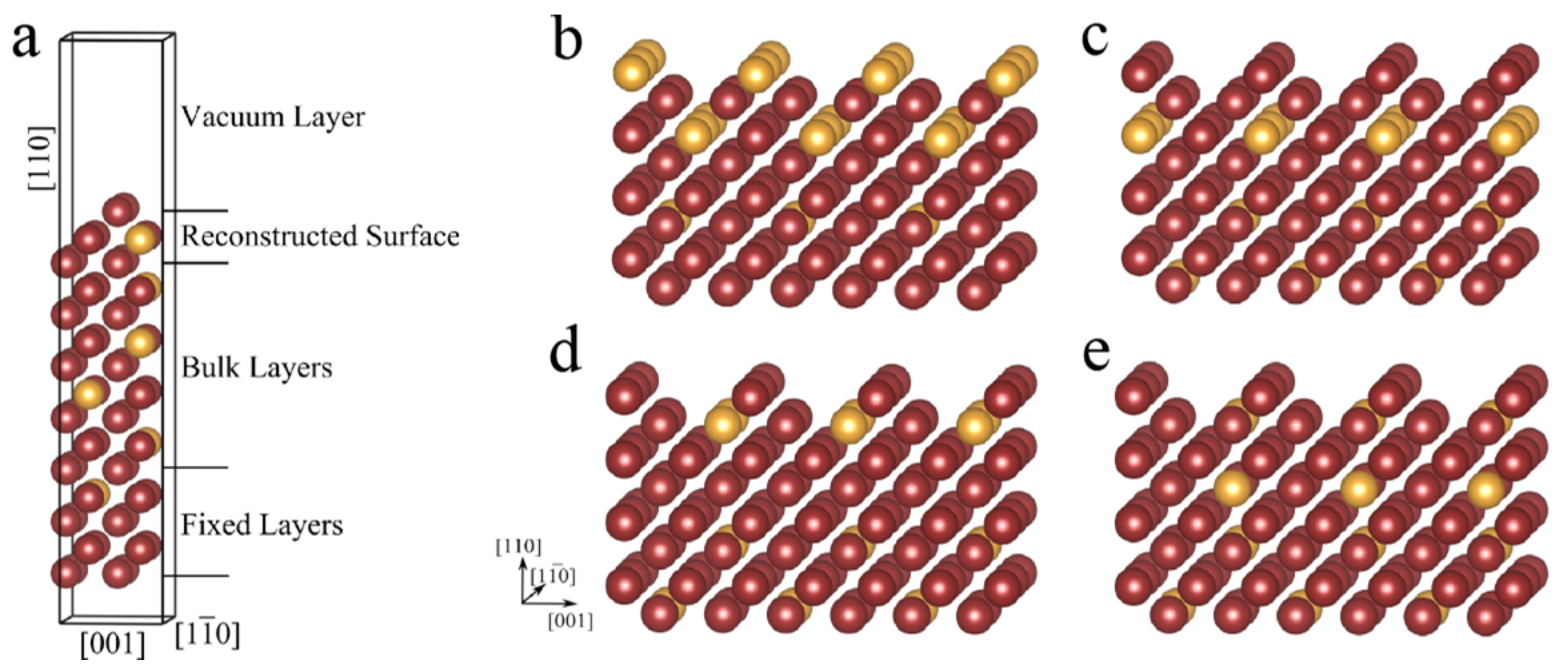

Extended Data Figure 4 | a, Simulation cell used in surface calculations. $\mathrm{Cu}$ and $\mathrm{Au}$ are presented by the brown and gold spheres respectively. b-d, $\mathrm{Cu}_{3} \mathrm{Au}$-type terminations of the reconstructed (110) surfaces of $\mathrm{Cu}_{90} \mathrm{Au}_{10}$ alloy. e, The reconstructed (110) surface of random $\mathrm{Cu}_{90} \mathrm{Au}_{10}$ alloy. 


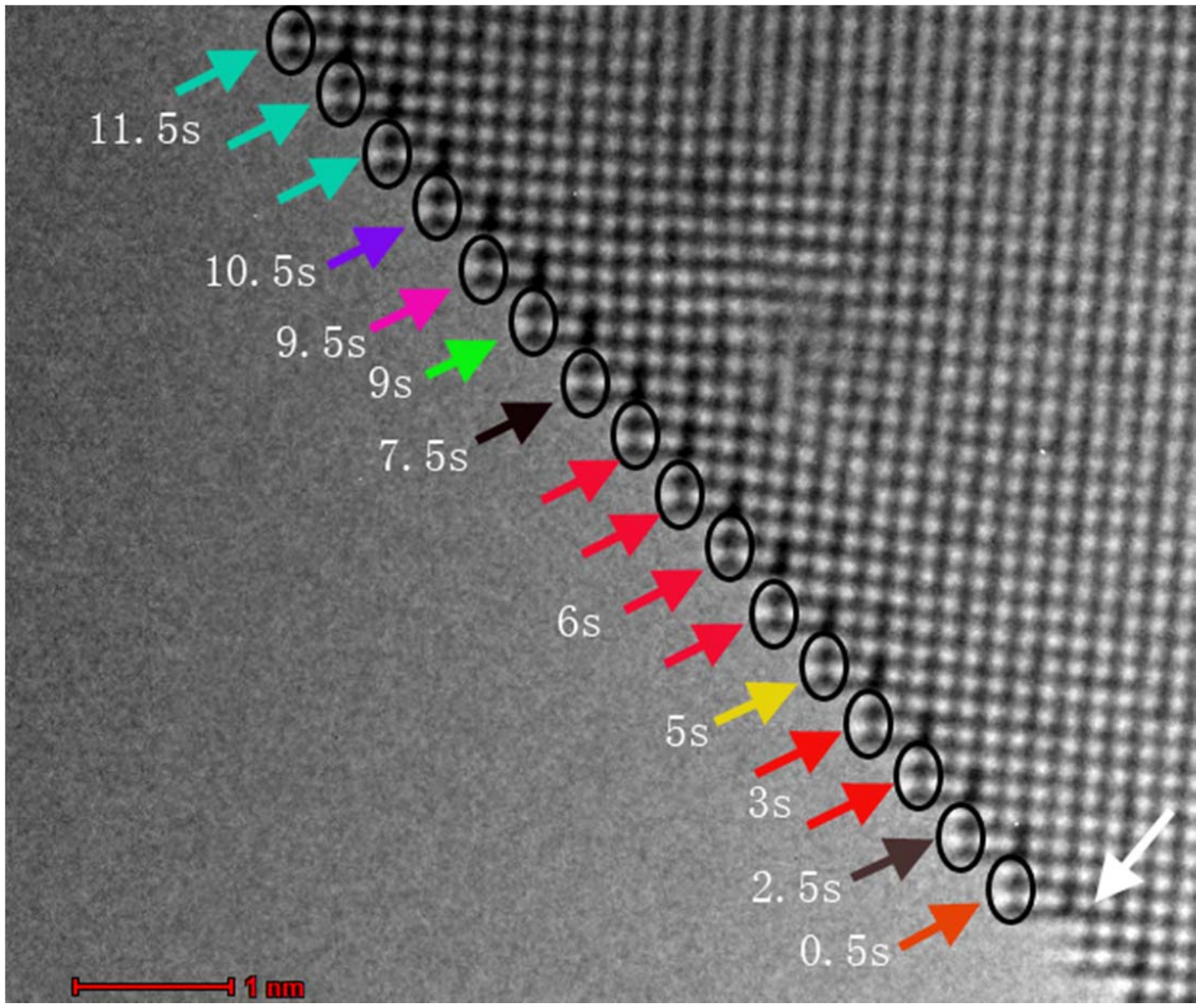

527 Extended Data Figure $5 \mid$ In-situ TEM observations of a $\{100\}$-type nano-faceted Au segregated (110)

528 surface at $350{ }^{\circ} \mathrm{C}$ and $1 \times 10^{-3}$ Torr of $\mathrm{H}_{2}$ gas flow show that surface atoms peel off as pairs of atomic columns from the (110) surface, where the arrows mark the locations and moment of time when the pair of surface atoms peel off from the (110) surface (see Supplemental in-situ TEM video 1). 

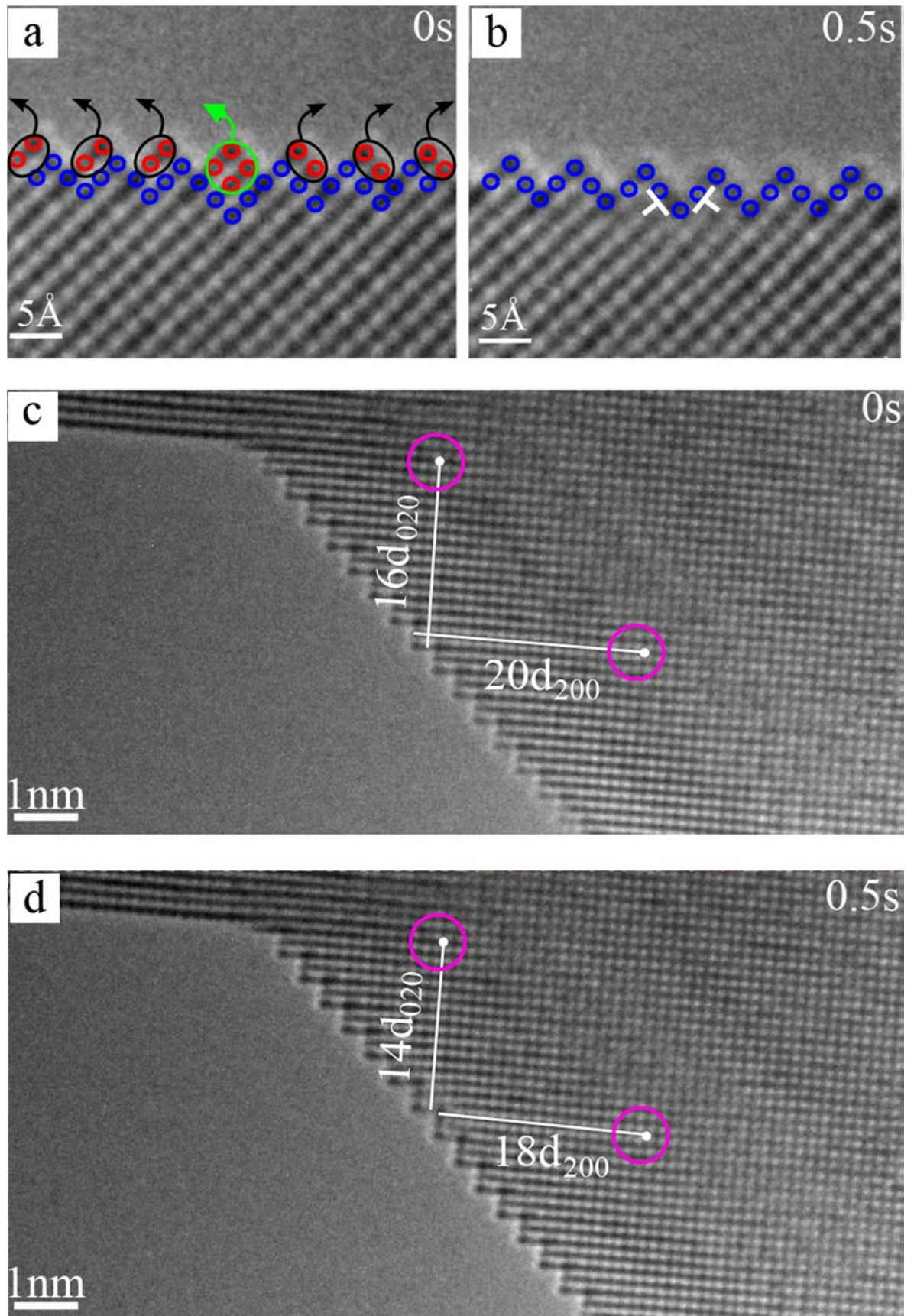

533 Extended Data Figure 6 | a-b, In-situ HRTEM images capturing the event of nucleating a misfit dislocation in a locally deepened trough formed by the departure of four columns of surface atoms in the region indicated by the large green circle, where the atoms diffusing away are denoted by small red circles and the atoms staying on the surface are denoted by small blue circles (see Supplemental in-situ TEM video 2). c-d, Identification of the misfit dislocation nucleation site shown in (a, b) by tracking the number of columns of surface atoms departing from the marked region by measuring the change in the distance between the outermost surface and the marked lattice points. 
542

543

544

545

546

547

548

549

550

551

552

553

554

555

556

557

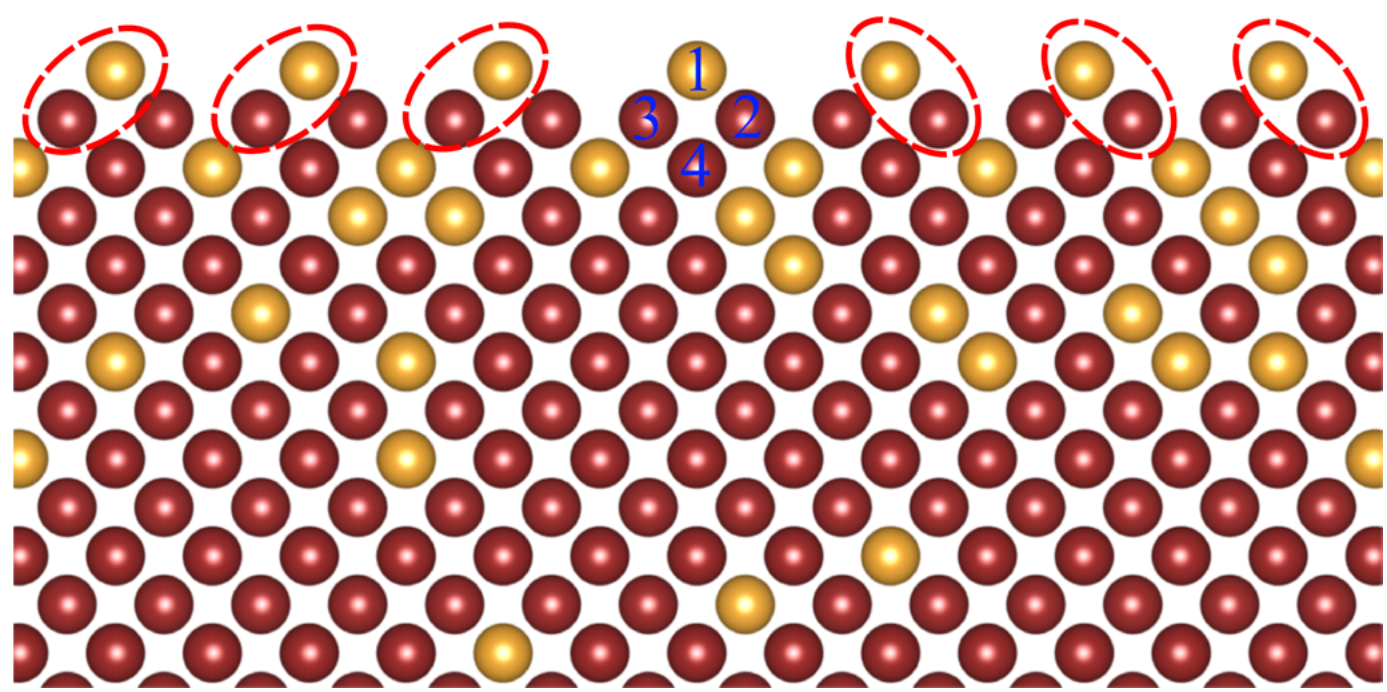

Extended Data Figure 7 | Structure model used for calculating the vacancy formation energy by sequentially removing surface atoms 1-4 from the trough-crest reconstructed surface of the $\mathrm{L}_{2}$ ordered $\mathrm{Cu}_{3} \mathrm{Au}$ segregated layer. The red dashed rings circle out the experimentally observed diatom detachment (see supplementary in-situ TEM videos 1 and 2, and Fig. 2). The energy cost for removing atoms 1-4 is calculated by DFT and listed in Extended data Table 1. 

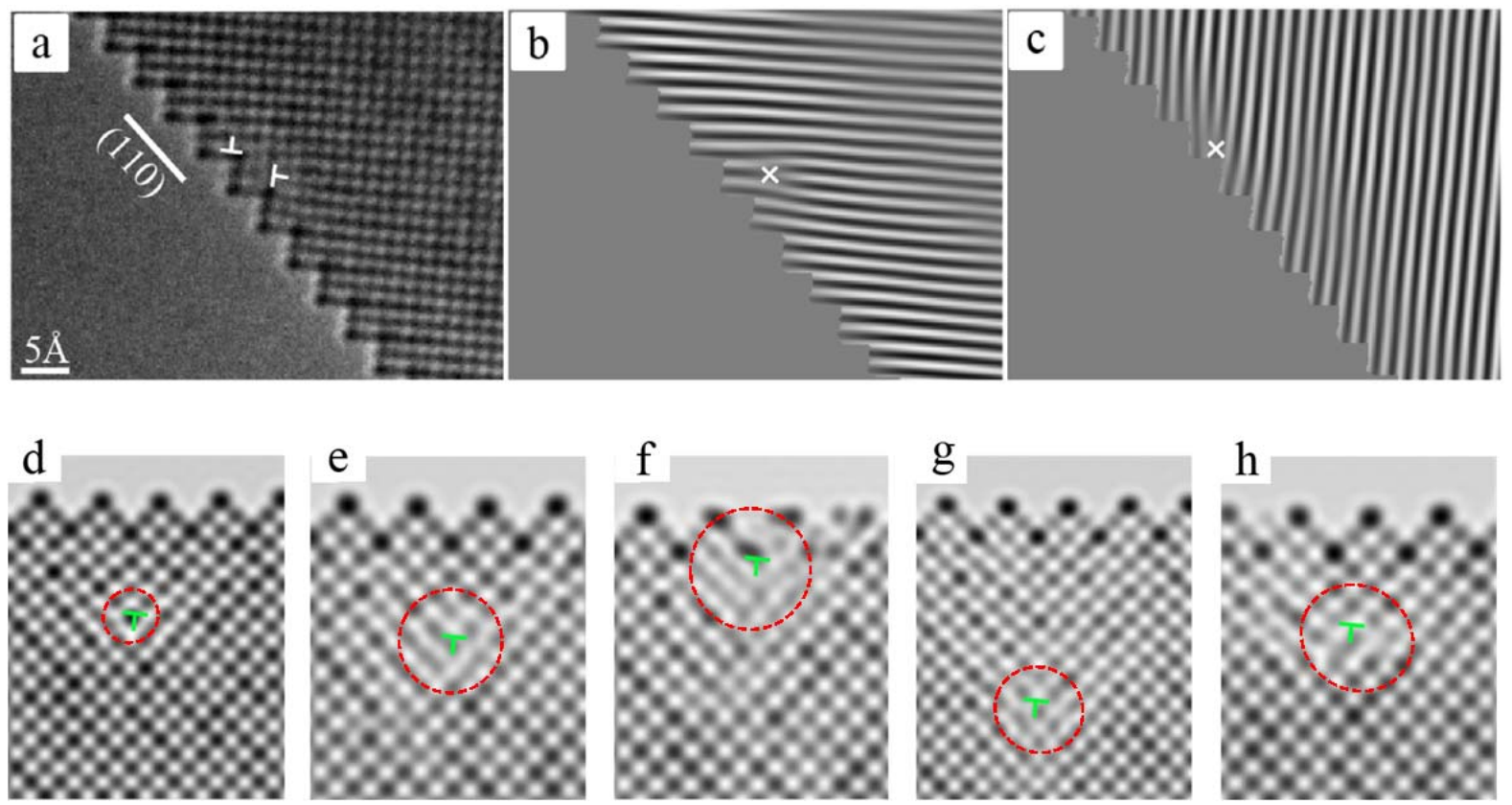

559 Extended Data Figure 8 | Identification of the location and property of dislocation cores by inverse

560 Fourier-filtered transform (IFFT) and HRTEM simulation. a, Raw HRTEM image showing the presence

561 of two dislocation cores residing along the $\mathrm{Cu}_{3} \mathrm{Au} / \mathrm{Cu}(\mathrm{Au})$ interface. $\mathbf{b}$, IFFT operation of the HRTEM using spot mask, white crossover marked the head position of horizontal misalignment, c, IFFT operation

563 of HRTEM using spot mask, white crossover marked the head position of vertical misalignment. d, the dislocation with the Burgers vector of $\mathrm{b}=\frac{1}{2} \mathrm{a}[\overline{1} 10]$ and dislocation line [001]. e-g, the dislocation with the

565 Burgers vector of $\mathrm{b}=\frac{1}{2} \mathrm{a}[\overline{1} 10]$ and dislocation line [111], [112] and [113], respectively. $\mathbf{h}$, the dislocation

566 with the Burgers vector of $\mathrm{b}=\frac{1}{2} \mathrm{a}[\overline{1} 12]$ and dislocation line [001]. 

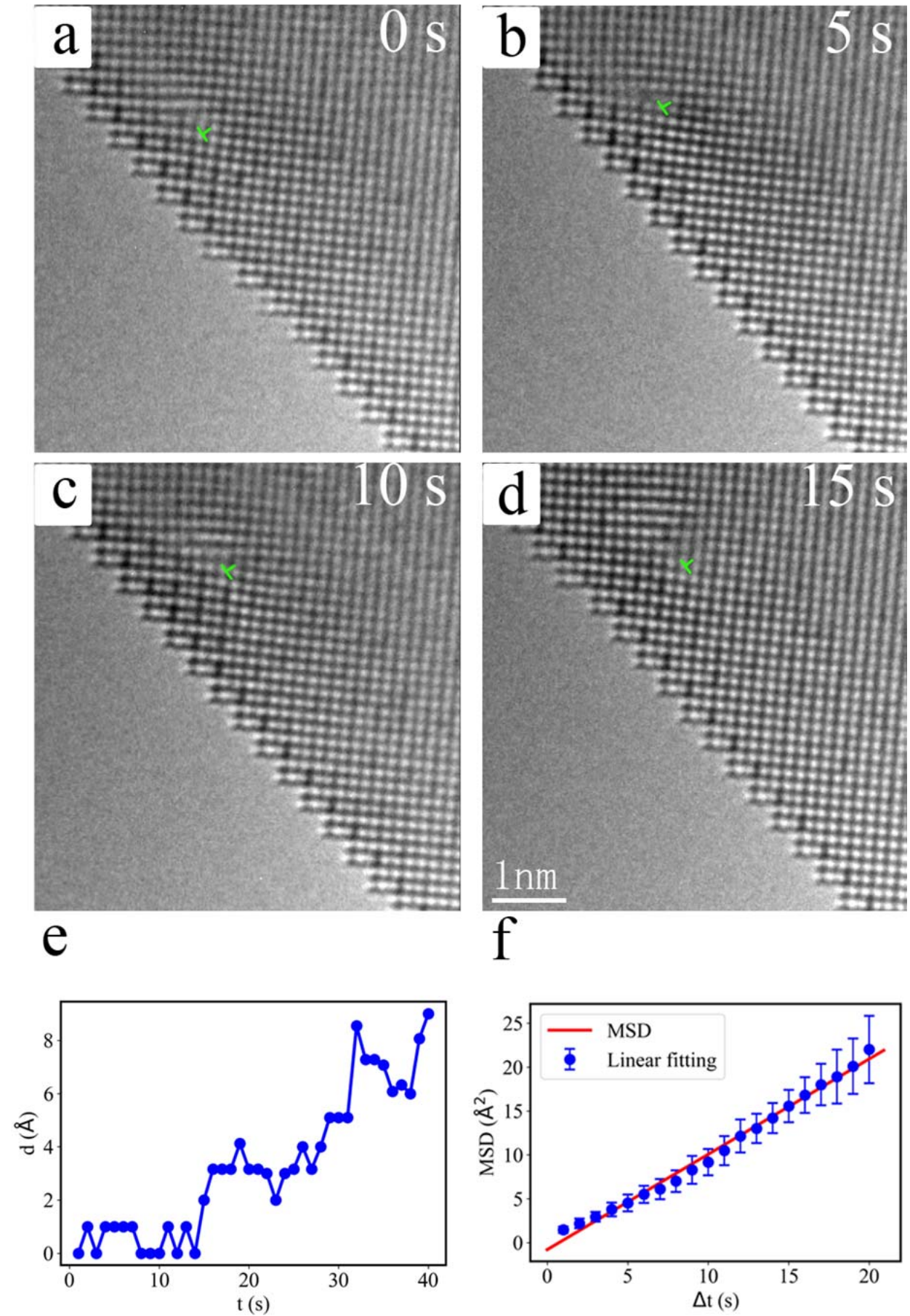

572 Extended Data Figure 9 a-d, In-situ HRTEM images showing the migration path of a sessile 573 dislocation (marked by the green "T") in the parent $\mathrm{Cu}(\mathrm{Au})$ region at $350{ }^{\circ} \mathrm{C}$ and $1 \times 10^{-3}$ Torr of $\mathrm{H}_{2}$ gas 574 flow (see Supplemental in-situ TEM video 6). e, Distance of the dislocation from its initial position as a 575 function of time. f, Mean square distance (MSD) versus time for the dislocation motion, where filled blue 576 squares represent experimental values measured from the in-situ TEM images and solid red line is the 577 linear fit. 
a

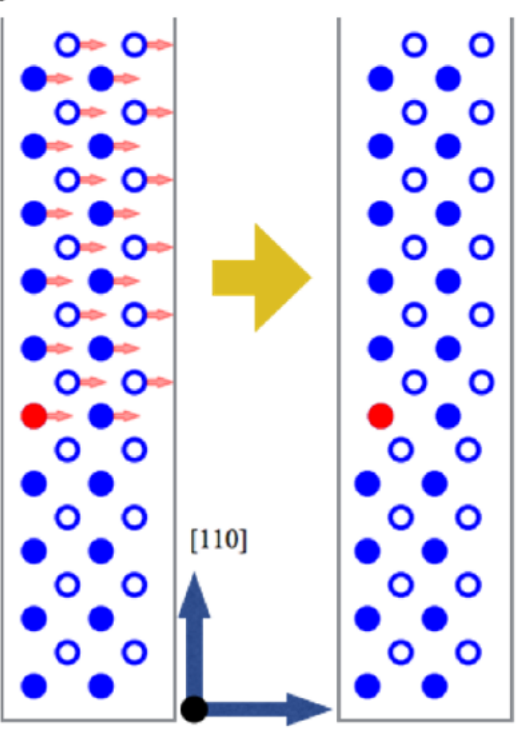

[001]

[110]

b

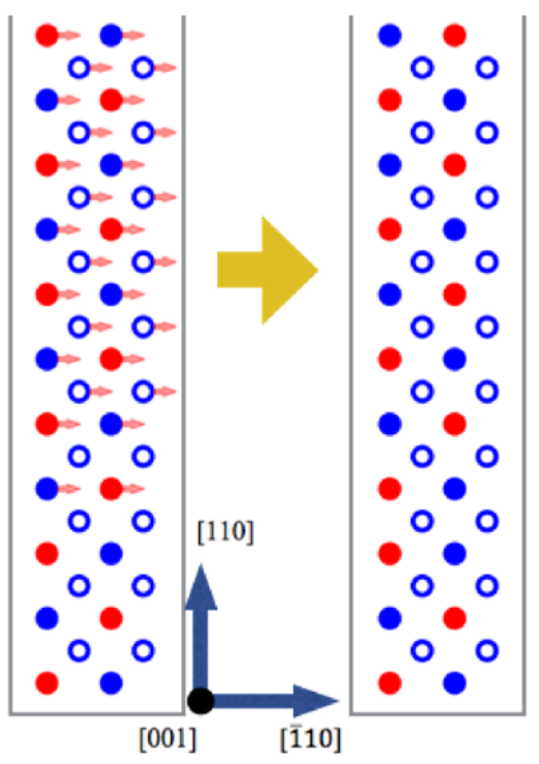

\section{c}

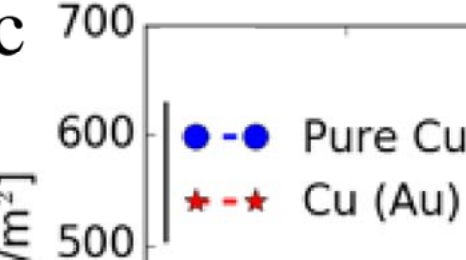

Extended Data Figure 10 | a, Sketch of the supercells to calculate the GSF in the $\mathrm{Cu}(\mathrm{Au})$ system, where the top (110) planes of pure $\mathrm{Cu}$ is shifted along the [110] direction, with one Au solute atom on the bottom layer nearest to the glide interface. b, Sketch of the supercells to calculate APB energies of L1 2 $\mathrm{Cu}_{3} \mathrm{Au}$, where top (110) planes of $\mathrm{Ll}_{2} \mathrm{Cu}_{3} \mathrm{Au}$ are shifted by $1 / 2$ [110]. In (a) and (b), blue color is for $\mathrm{Cu}$ and red color is for $\mathrm{Au}$. The filled circles and unfilled circles indicate different atom coordinates along the projection direction perpendicular to the screen. c, GSF in pure $\mathrm{Cu}$ and $\mathrm{Cu}(\mathrm{Au})$ systems calculated from supercells in (a). 

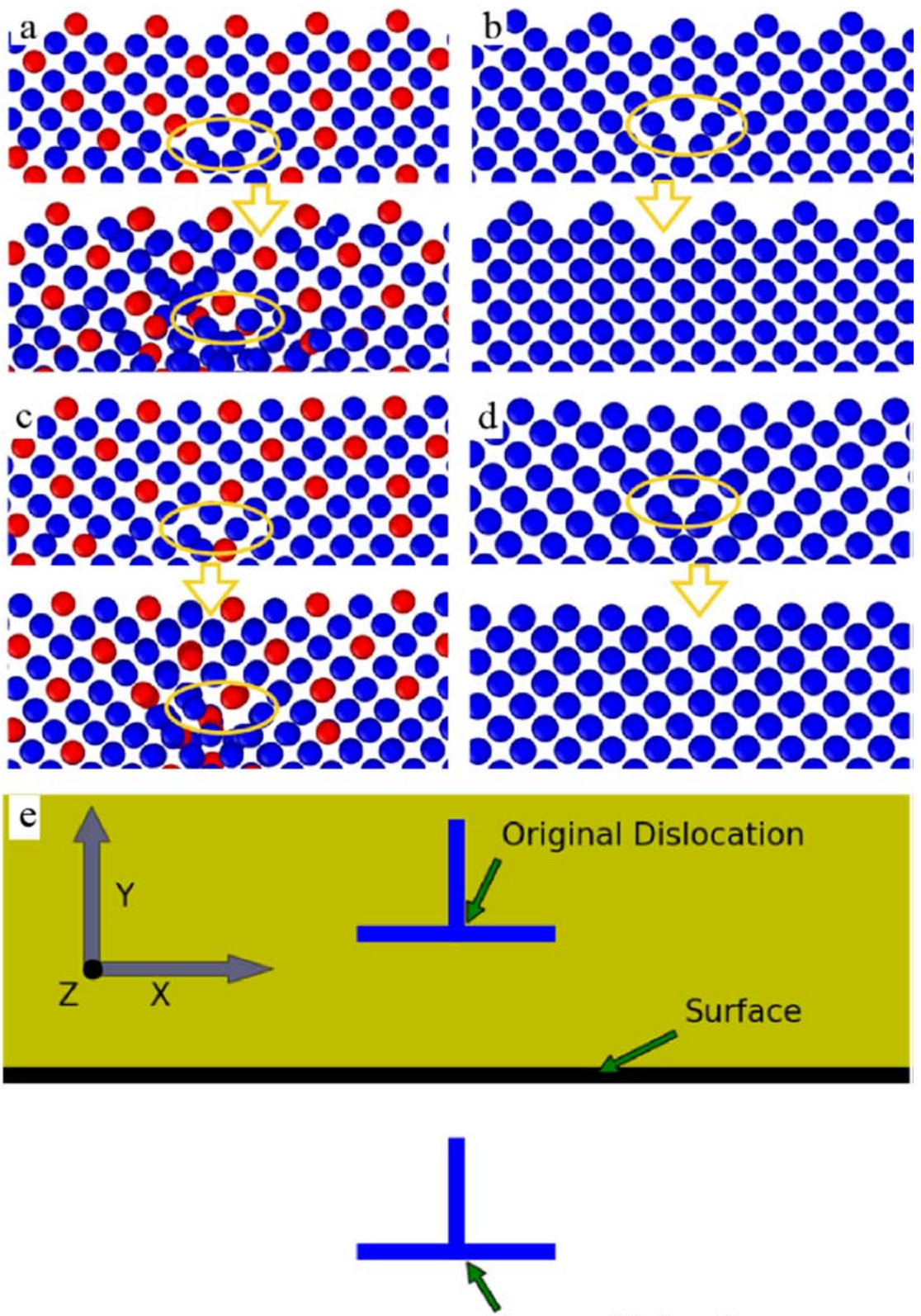

Image Dislocation

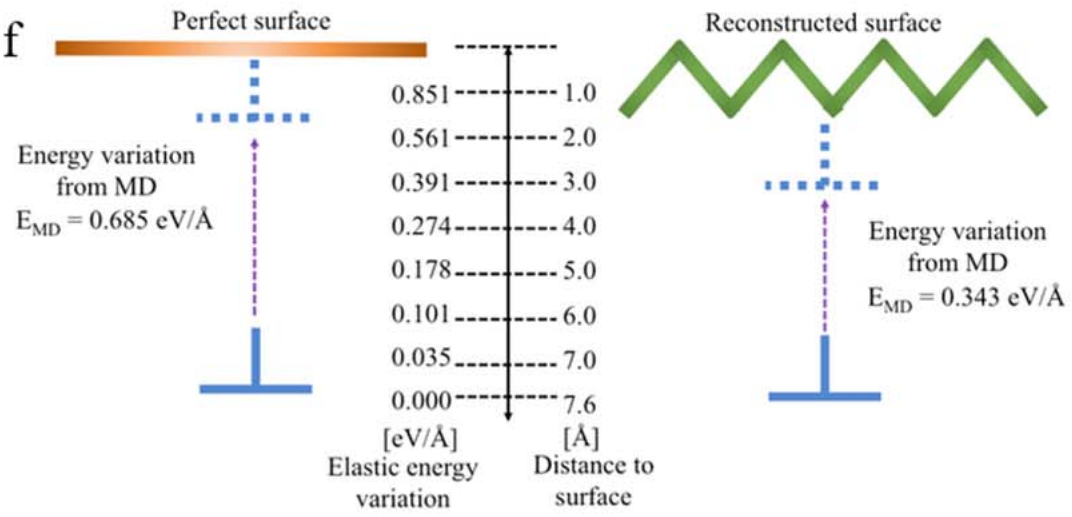


588

589

590

591

592

593

594

595

596

597

598

599

600

601

602

603

604

605

606

607

608

609

610

611

612

613

614

615

616

617

618

619

620

621

622

623

624

625

Extended Data Figure $11 \mid 1 / 2[\overline{\mathbf{1}} 10](110)$ dislocation behavior near the surface layers of $\mathrm{L1}_{2} \mathrm{Cu}_{3} \mathrm{Au}$ and $\mathbf{L 1}_{2} \mathrm{Cu}_{3} \mathbf{A g}$. a, $\frac{1}{2} a[\overline{1} 10](110)$ edge dislocation glides along the $\mathrm{Cu}_{3} \mathrm{Au} / \mathrm{Cu}(\mathrm{Au})$ interface. b, the $\frac{1}{2} a[\overline{1} 10](110)$ dislocation in pure $\mathrm{Cu}$ annihilates on the free surface by diffusionless transformation. (a) and (b) are calculated using the $\mathrm{CuAu}$ potential. c, The $\frac{1}{2} a[\overline{1} 10](110)$ edge dislocation glides along the $\mathrm{Cu}_{3} \mathrm{Ag} / \mathrm{Cu}(\mathrm{Ag})$ interface. $\mathbf{d}$, The $\frac{1}{2} a[\overline{1} 10](110)$ in pure $\mathrm{Cu}$ annihilates on the free surface by diffusionless transformation. (c) and (d) are calculated using the $\mathrm{Cu} / \mathrm{Ag}$ EAM potential. e, Sketch of the image dislocation of $1 / 2[\overline{1} 10]$ (111) edge dislocation near the free surface. $\mathbf{f}$, Comparisons of energy variations for dislocation annihilation from pure elastic contribution according to Eq. 20 in Extended Data and those from direct MD simulations shown in (b) and (d). 

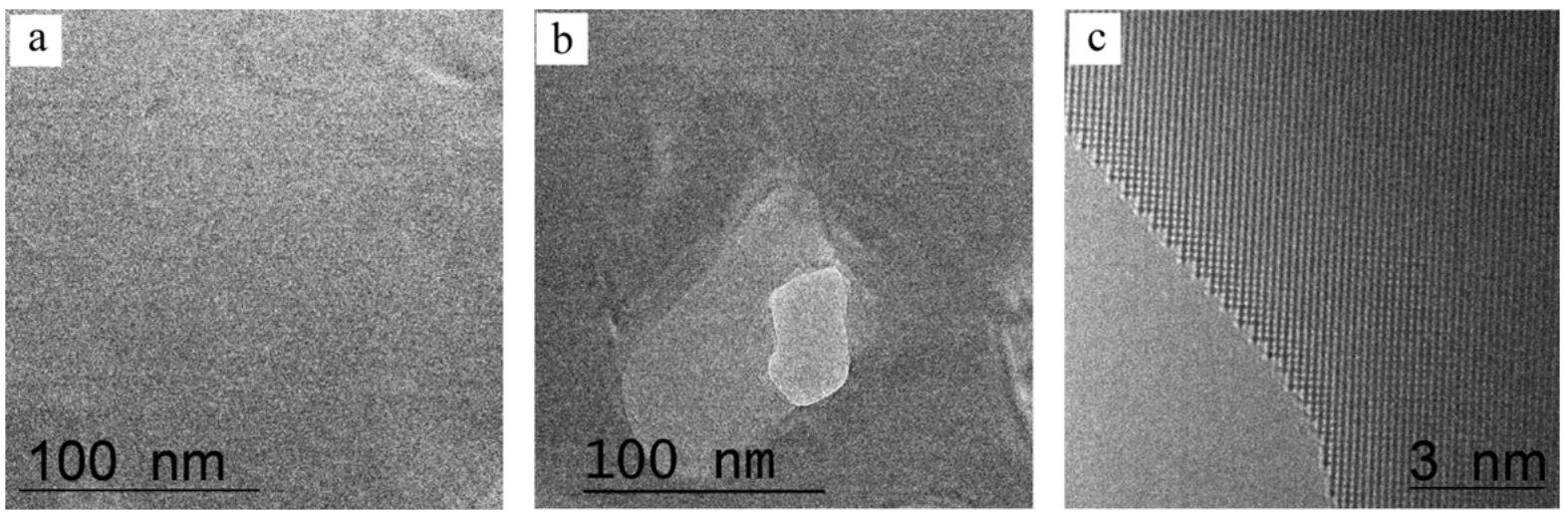

Extended Data Figure 12 Possible electron beam irradiation effect on the surface structure evolution. a, the starting continuous $\mathrm{Cu}_{90} \mathrm{Au}_{10}(100)$ thin film. $\mathbf{b}$, Nanopore formation after $\sim 20 \mathrm{~min}$ of annealing at 350 ${ }^{\circ} \mathrm{C}$ and 0.001 Torr of $\mathrm{H}_{2}$ gas flow. To avoid any possible electron beam irradiation effect, the e-beam was blanked off during the annealing process. c, HRTEM image showing the formation of the characteristic $\mathrm{Au}$-segregation induced trough-crest surface reconstruction of the $\mathrm{Cu}_{3} \mathrm{Au}$ surface layer after the e-beam was un-blanked for TEM imaging. 
634

635

636

637

638

639

640

641

642

643

644
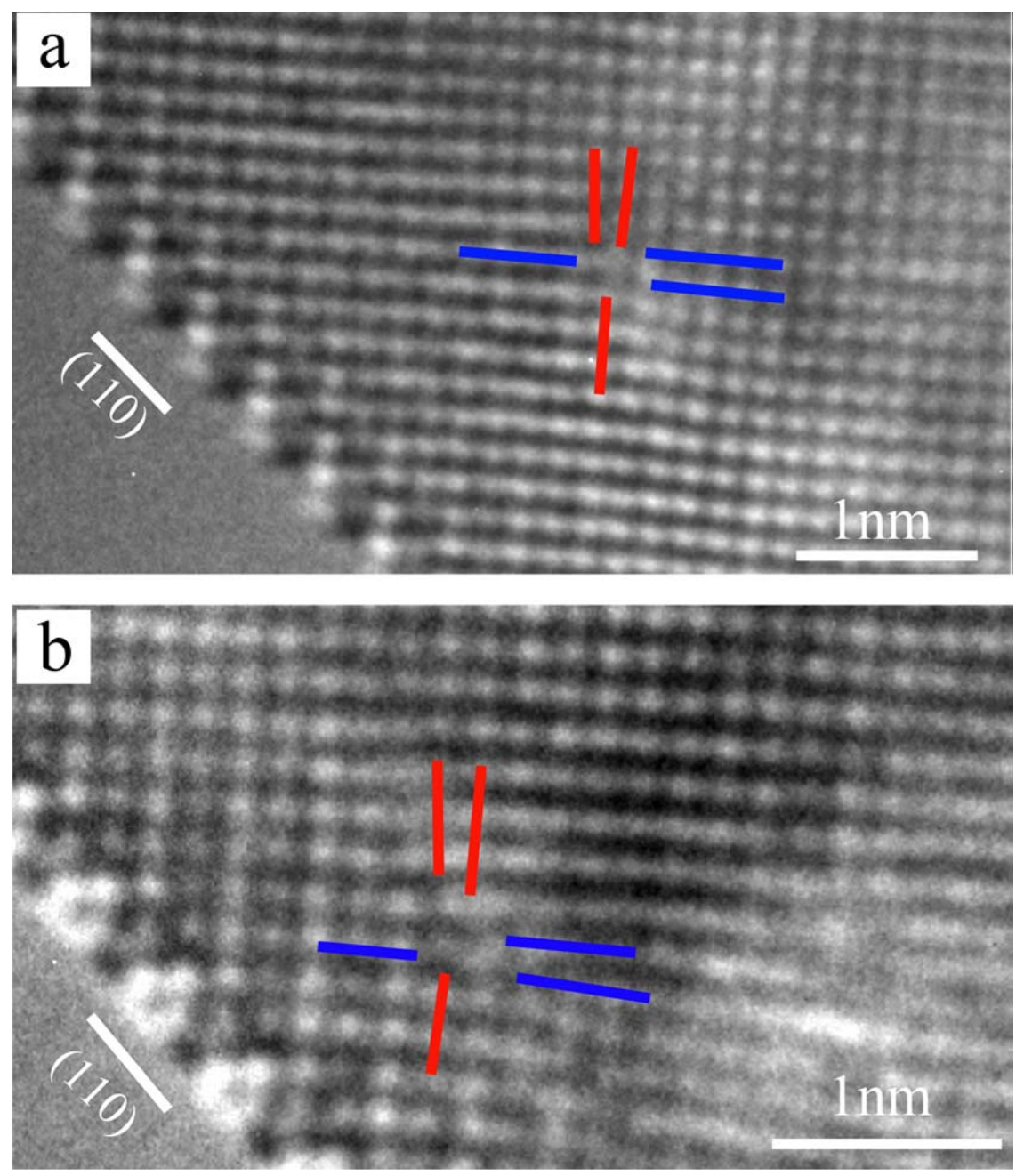

Extended Data Figure 13 | Dislocation motion with blanked electron beam. a, $\frac{1}{2} \mathrm{a}[\overline{1} 10](110)$-type dislocation located in a $\mathrm{Cu}_{90} \mathrm{Au}_{10}(100)$ film annealed at $350{ }^{\circ} \mathrm{C}$ and $1 \times 10^{-3}$ Torr of $\mathrm{H}_{2}$ gas flow. b. The ebeam was blanked off for a few seconds and then un-blanked for TEM imaging, showing that the dislocation has undergone some migration by moving toward the surface side by a few atomic spacings without the e-beam irradiation. 

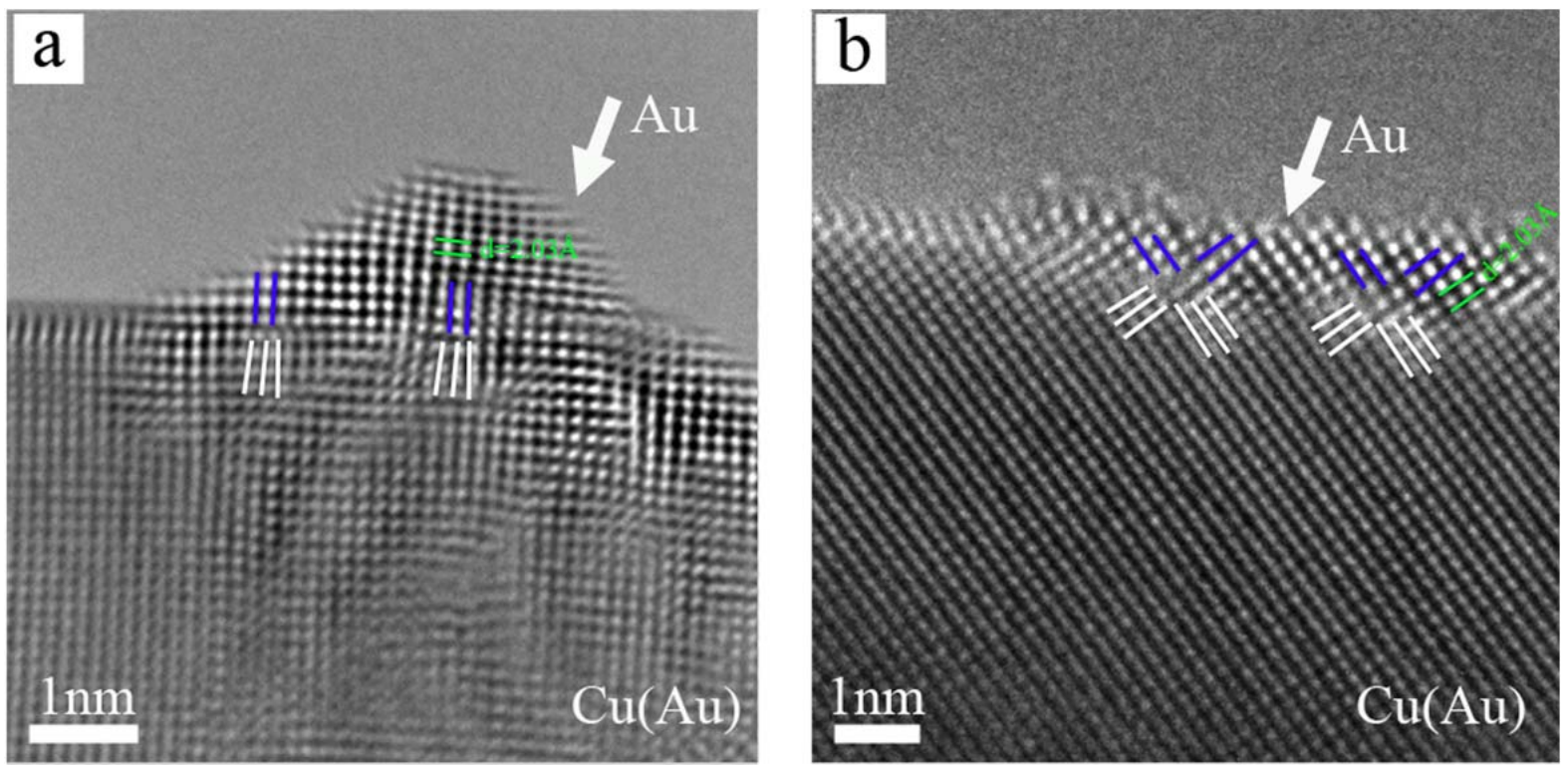

646 Extended Data Figure 14 HRTEM images of the Au surface segregation induced Au clusters on the 647 (100) and (110) surfaces at $600{ }^{\circ} \mathrm{C}$ and $\mathrm{pH}_{2}=1 \times 10^{-3}$ Torr. a, misfit dislocation array formed along the 648 [100] direction of the $\mathrm{Au} / \mathrm{Cu}(\mathrm{Au})$ interface. $\mathbf{b}$, misfit dislocation array along the [110] direction of the $649 \mathrm{Au} / \mathrm{Cu}(\mathrm{Au})$ interface. The lattice mismatch at the $\mathrm{Au} / \mathrm{Cu}(\mathrm{Au})$ interface is marked by the blue and white 650 line.

651

652

653 